\title{
EL ESTAdO CHILENO Y LA PROTECCION A LA INFANCIA
}

\author{
Por el Dr. GUILlermo MORALES beLTraMl \\ Director General de Protección a la Infancia y Adolescencia.
}

Este trabajo tiene por objeto dar a conocer a los pediatras americanos que se reunirán en Montevideo durante los primeros días de diciembre próximo en los Congresos de Pediatria, cuál ha sido la preocupación del Estado chileno en materia de protección y asistencia de la infancia, para cuyo objeto analizaremos las leyes, reglamentos orgánicos y demás iniciativas estatales más destacadas sobre el tema.

Es nuestro propósito hacer una especie de balance de los resultados obtenidos, a la luz de los datos que nos proporcione la experiencia adquirida en la aplicación práctica de las disposiciones proteccionales aludidas y destacar, en seguida, cuáles serán las medidas que es necesario auspiciar, modificar o encauzar mejor en el futuro.

Por estos mismos motivos esta ponencia deberá revestir caracteres amplios y muy generales, sin entrar en detalles que la alarguen inútilmente $\mathrm{y}$ confundan su comprensión justa y proporcionada.

Puede afirmarse que, hasta 1928, la asistencia del niño chileno estuvo entregada a la bondad y generosidad de la beneficencia privada, siendo intrascendentes y esporádicas las medidas administrativas dictadas por los gobiernos para solucionar alguno de los numerosos aspectos que el problema presentaba en el pais.

No es aventurado afirmar que dos importantes aspectos comenzaban ya a alarmar a la opinión pública y a los poderes del Estado: eran ellos la alta mortalidad infantil, determinada por una serie de circunstancias biológicas y socia- les que escapaban a la posibilidad de solución que podían darie las instituciones particulares y la medicina curativa de los hospitales y policlínicas de niños y la irregularidad de los menores pre-escolares, escolares y adolescentes que iba en aumento, con sus espectaculares expresiones: vagancia, mendicidad, abandono de hogar y delincuencia consecuencial.

El primero de estos problemas aparecía de responsabilidad eminentemente médica: se multiplicaban los servicios de Gotas de leche y consultorios de lactantes, se debatía ampliamente el asunto en la Sociedad de Pediatría, pero las cifras de mortalidad, lejos de disminuir, se alzaban en franco aumento.

El segundo fenómeno social era la preocupación diaria de la prensa: se pedía a las autoridades, a los abogados, a los legisladores, que adoptaran medidas eficaces para reprimir estos síntomas, que, aunque inequívocos de la baja del standard de vida del pueblio chileno, se atribuían a la falta de medidas represivas para ahogarlos.

Fué así, pues, cómo después de laboriosos estudios, con la participación de médicos y abogados, se dictó la Ley $\mathrm{N}^{Q}$ 4,447, llamada de Protección de Menores, en el año 1928.

\section{La Ley de Menores.}

La doctrina matriz y el espiritu fundamental de esta Ley se inspiran en el propósito de legislar en bien del niño con una conciencia proteccional y tutelar, antes que punitiva o represiva. 
de 1928 hasta la fecha. Expone en detalle las disposiciones de la Ley de Menores $\mathrm{N}^{\circ} 4,447$, base fundamental de la organización de los servicios estatales y de las normas juridicas que rigen la protección a la infancia.

En párrafos apartes analiza las disposiciones pertinentes a la infancia, del Código Sanitario, de la Ley de Seguró Obrero, del Código del Trabajo. Se refiere también a la importancia y a la utilidad pública de los servicios de Ia Beneficencia.

Finalmente, detalla las funciones que incumben a la Dirección General de Protección a la Infancia, servicio que constituye el primer ensayo de fusión de todas las reparticiones puramente estatales destinađas a la atención del niño.

Propone, finalmente la formación del Fondo Nacional de Protección a la Infancia, como manera de solventar los ingentes gastos que requiere la organización de una protección materno-infantil amplia e integral.

\section{Summary.}

The author considers the different initiatives developped by the Chilean Government in matters regarding infantile welfare since 1928 up to present date. He explains in detail the Law of Minors number 4447 , fundamental in the organization of the government services and laws that control infantile protection.

Afterwards he analyzes the different dispositions concerning infancy in the Sanitary Code, Labor Code and Workmen's Security Law. He refers also to the importance of the benefits of private welfare.

Finally, he details the different functions that concern the Central Commitee of Protection of Infancy, that constitutes the first trial of fusion of all the government agencies concerned in the child's care.

He proposes, finally, the formation of a National Fund for Protection of Infancy as a mean of financing the organization of an ample plan of maternal and infantile protection. 